\title{
INVESTIGATION OF MEGAWATT MAGNETRON INJECTION GUNS WITH SECONDARY-EMISSION CATHODES
}

\author{
V. V. Zakutin, A. N. Dovbnya, N. G. Reshetnyak, Yu. Ya. Volkolupov, and M. A. Krasnogolovets, \\ Kharkov Institute of Physics and Uskoritel' Research Complex Technology, National Scientific \\ Center, Akademicheskaya ul. 1, Kharkov, 61108 Ukraine
}

\section{INTRODUCTION}

Recent years have seen extensive research into coldcathode magnetron injection guns of ordinary [1-6] and inverted [6, 7] type. Such sources employ secondary emission in crossed electric and magnetic fields. Among their merits are long operating life, high current density, relatively simple configuration, the capability of producing hollow beams, etc. Therefore, they could make emitters for long-lived high-power microwave generators [3] or fast highvoltage devices [8]. Offering intense electron beams, these guns enable one to achieve high levels of pulse power when used in klystrons or other microwave tubes.

\section{EXPERIMENTAL SETUP}

Experiments to generate high-power electron beams with magnetron injection guns using secondary-emission cathodes (SECs) were conducted in the apparatus schematically shown in Fig. 1. A magnetron injection gun is driven by modulator 7 , which produces 4 - to $100-\mathrm{kV}$ negative voltage pulses of width 2-10 us and repetition rate 10-50 Hz. The pulses are applied to copper cathode 5, whereas anode 6 is grounded via resistor $R_{3}$. The anode is made of stainless steel or copper. Two alternative techniques to initiate secondary emission were employed. They respectively use a pulse from external generator 2 or a specially generated overshoot in a cathode voltage pulse. Secondary emission takes place in the trailing edge of the initiating pulse or overshoot. A magnetic field to produce and transport a beam is created by solenoid 4. The beam current is measured with Faraday cup 7 and resistor $R_{4}$; cathode voltage, via divider $R i \sim R_{2}{ }^{\prime}$, ${ }^{a n} \mathrm{~d}$ beam size, with the help of a print on an X-ray film or a molybdenum foil. The gun is situated in stainless-steel vacuum chamber $3 \mathrm{kept}$ at a pressure of about $10^{\prime \prime}$ torr.

The magnetic field was created by two methods. One of them generates a pulsed field when a capacitor is discharged through the solenoid [9]. The other provides a static field: a constant current is passed through the solenoid, which then requires water cooling [10]. Curves 1 and 2 in Fig. 2 depict the distributions of the magnetic field on the solenoid axis for the two cases, with $A, C$, and $F C$ standing for the anode, cathode, and Faraday cup, respectively.

The pulsed approach (Fig. 2, curve 1) provides magnetic fields of considerable strength (up to 5000-6000 Oe) and longitudinal uniformity (within $\pm 5 \%$ ). It is also useful for transporting a beam through the resonators of a microwave tube. On the other hand, the pulse rate here is limited by the actual designs of the storage capacitor and the switch. Furthermore, the longitudinal pulsed field distribution may alter due to the attenuation of the field as it diffuses through the walls of the resonators and the vacuum chamber (see, e.g., $[9,11])$. Finally, note that contaminants enter the cathode-anode gap owing to electron bombardment and deposition on the cathode surface during time intervals between the high-voltage pulses, so that vacuum breakdown of the gap may occur if the pulse rate is too low [12].

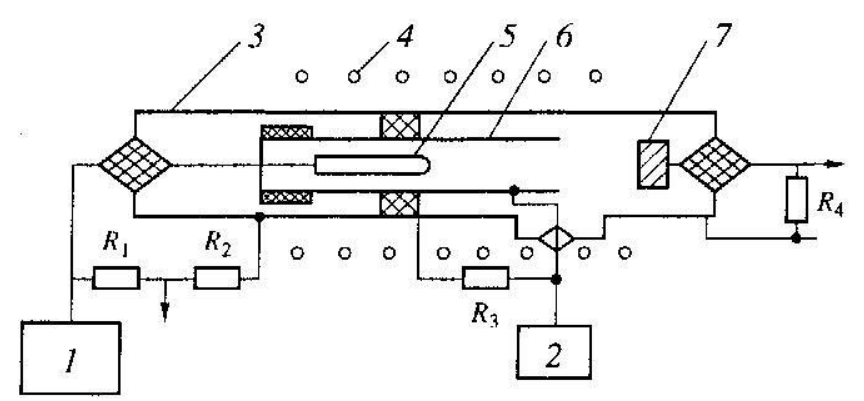

Fig. 1.

With the static approach, the solenoid consumes more power and needs water cooling. The magnetic field is uniform in a shorter interval (curve 2 in Fig. 2). Finally, the instability of the coil current has to be taken into account, so that the rate of drive pulses from the modulator must be adjusted to the mains frequency.

\section{RESULTS AND DISCUSSION}

First, we tested a gun with a copper cathode of diameter $40 \mathrm{~mm}$ and a stainless-steel anode of diameter $78 \mathrm{~mm}$ and length about $100 \mathrm{~mm}$. Secondary emission was initiated by overshoots (up to $160 \mathrm{kV}$ ) in cathode pulses. Figure 3 shows the beam current at the Faraday cup versus the cathode pulse height. It is seen that the current obeys the Child-Langmuir law. For each value of the cathode voltage, the magnetic field was adjusted so as to maximize the beam current. We achieved stable generation of an electron beam with a current close to 50 $\mathrm{A}$ at a voltage of $100 \mathrm{kV}$ and a magnetic field of about 
1600 Oe. Accordingly, the microperveance and the beam power approximated to 1.6 and $5 \mathrm{MW}$, respectively. The measurements revealed that the gun endures $120 \mathrm{kV}$, which allows one to produce up to 8 MW of beam power.

Then, we tested a gun with a copper cathode of diameter $40 \mathrm{~mm}$ and a stainless-steel anode of diameter $50 \mathrm{~mm}$ and length about $100 \mathrm{~mm}$. It generated a beam current of $50 \mathrm{~A}$ at $30 \mathrm{kV}$ and 2200 Oe, with the microperveance and the beam power being close to 10 and $1.5 \mathrm{MW}$, respectively.

These results are similar to those reported in [6], where experiments were performed on a gun made of $\mathrm{BeCu}$ and stainless steel with (1) the cathode diameter $50 \mathrm{~mm}$ and anode diameter $60 \mathrm{~mm}$ (ordinary type) and (2) cathode diameter $54 \mathrm{~mm}$ and anode diameter $43 \mathrm{~mm}$ (inverted type). A beam current of about $100 \mathrm{~A}$ was obtained at $40 \mathrm{kV}$ and about 1800 Oe. The beam power and the microperveance were close to $4 \mathrm{MW}$ and 12 , respectively.

Thus, SEC magnetron injection guns provide high beam powers. However, their use in microwave electronics is hindered by large values of microperveance and the outer beam diameter. (The latter was reported to vary between 45 and $55 \mathrm{~mm}$, depending on the experimental setup.)

Another way to increase the beam power is the use of several (six or more) guns connected in parallel. This may yield a high total current and power even if each gun has moderate beam current, power, microperveance, and beam size.

Admittedly, such an approach may give rise to the same difficulties as the parallel connection of switches. Once one of the guns has started generating, the cathode pulse heights of other guns may fall to impermissi-bly small values (the guns do not generate). Also, a multigun configuration may, in principle, have a decreased breakdown voltage, since it varies inversely as $S^{0}{ }_{-}^{1}$, where $S$ is the total area of the electrodes.

$H$, Oe

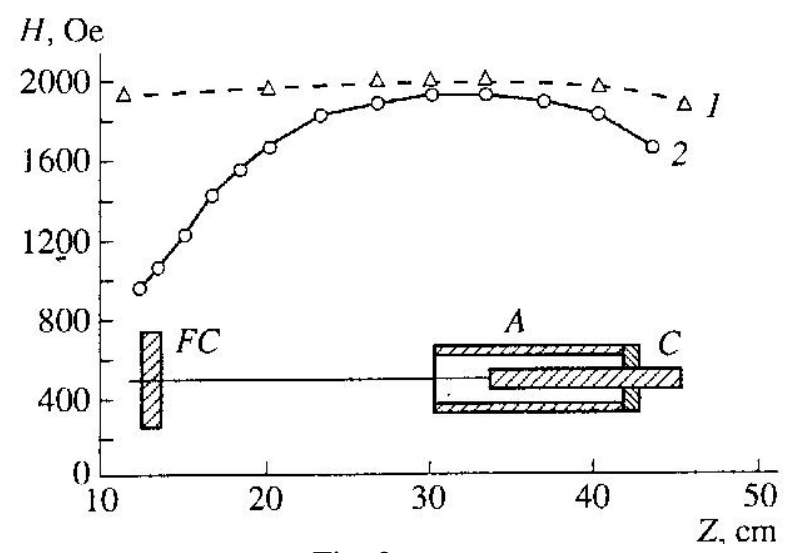

Fig. 2.
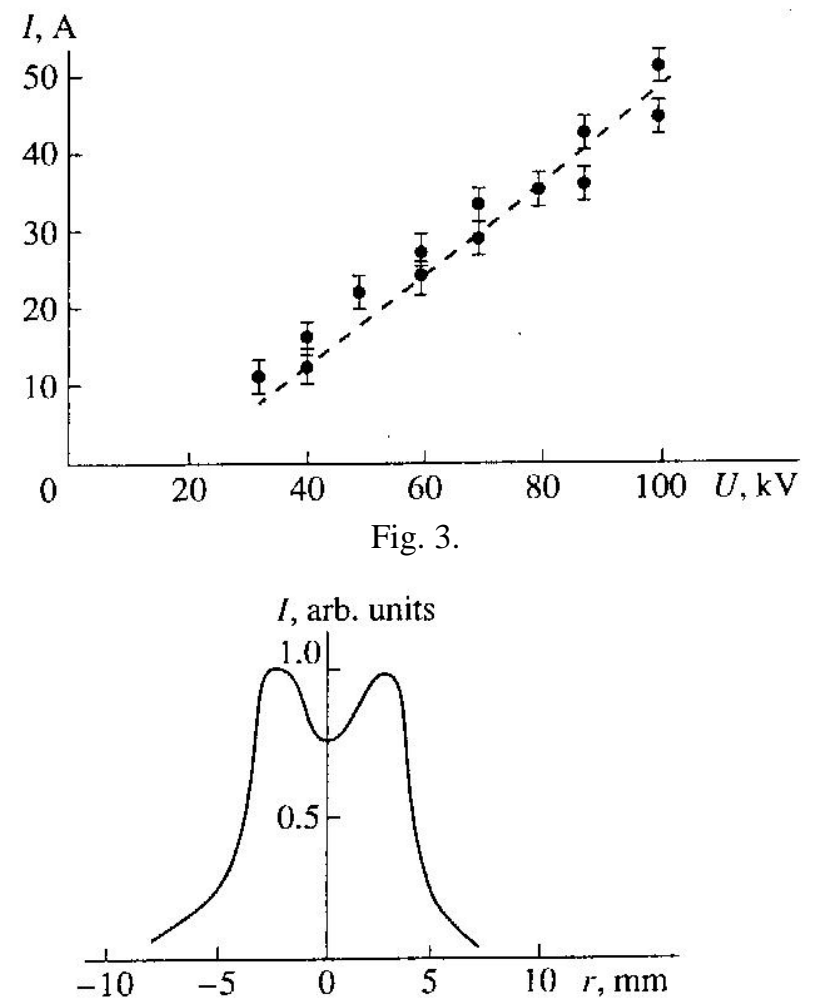

Fig.4.

With a fairly large number of guns (six and even more), the decrease in breakdown voltage is insignificant (about $10-20 \%$ ). So may be that in the cathode pulse height if the modulator has a small output resistance. In our experiments, the pulse height decreased by less than $30 \%$ for a modulator resistance of $2000 \mathrm{Q}$. Note that a beam is generated if the height of the cathode pulses varies to within $40 \%$, as was reported in [13]. Furthermore, it was shown that the cathode voltage decreases appreciably after a few nanoseconds. On the other hand, the time to create an electron layer in an SEC magnetron injection gun, and hence the rise time of the pulsed beam current, can be reduced to 2 ns [14]. Thus, it is possible to meet the challenge of generating electron beams in multigun systems.

We tested systems with six and eight guns. In the former case, the guns were arranged in a circle of diameter about $60 \mathrm{~mm}$, with the cathode and the anode diameter being 5 and $26 \mathrm{~mm}$, respectively. Six beams were produced, each having an outer diameter of about $9 \mathrm{~mm}$. At a cathode voltage of $40 \mathrm{kV}$ and a magnetic field close to $2000 \mathrm{Oe}$, each gun delivered 18 A of beam current with a microperveance of about 2.5. The total beam current and power approximated to $100 \mathrm{~A}$ and $4 \mathrm{MW}$, respectively. In the latter case, the guns were arranged in a circle of diameter about $70 \mathrm{~mm}$. The cathode and the anode diameter were 5 and $22 \mathrm{~mm}$, respectively. Eight beams were produced, each having an outer diameter and a microperveance of about $9 \mathrm{~mm}$ and 2, respectively. The total beam current and power approximated to $60 \mathrm{~A}$ and 2 MW for the cathode voltage and the magnetic field 
close to $30 \mathrm{kV}$ and 2000 Oe. Research is under way to obtain a beam power up to $10 \mathrm{MW}$.

With a pulsed magnetic field (curve / in Fig. 2), a beam was transported for a distance of 50-100 mm from the anode. With a static field (curve 2 in Fig. 2), the distance was 50 and $180 \mathrm{~mm}$. For both cases, the beam cross section at the collector was an annulus whose inner diameter is approximately equal to the cathode diameter and whose width is $1-1.3 \mathrm{~mm}$. The azimuthal intensity distribution was uniform over the annulus. Figure 4 shows the radial intensity distribution at a distance of $50 \mathrm{~mm}$ from the anode when the cathode and anode diameters are 5 and 26 $\mathrm{mm}$, respectively. It is seen that the outer beam diameter is less than $10 \mathrm{~mm}$. The magnetic field near the X-ray film is found to be lower by a factor of about 1.8 compared with that at the gun, as suggested by curve 2 in Fig. 2 . Nevertheless, the beam size remains almost the same. The above results indicate that the magnetic field holds the beam together and that its attenuation does not cause beam spreading. These findings are relevant to transporting and injecting electron beams.

\section{CONCLUSIONS}

The experiments have demonstrated that single SEC magnetron injection guns and their combinations can produce electron beams of considerable power. Thus, the guns could serve as electron sources in highpower microwave tubes.

\section{REFERENCES}

1. J. F. Skowron, Proc. IEEE 61 (3), 330 (1973).

2. S. A. Cherenshchikov, Elektron. Tekh., Ser. 1, No. 6, 20 (1973).

3. A. N. Dovbnya, V. V. Zakutin, V. F. Zhiglo, et al, in Proceedings of the 5th European Panicle Accelerators Conference, Sitges, 1996, Ed. by S. Myers, A. Pacheco, R. Pascual, et al. (Inst. of Physics Publ., Bristol, 1996), Vol. 2, p. 1508.

4. A. N. Dovbnya, V. V. Mitrochenko, et al., in Proceedings of the 1997 Particle Accelerators Conference, Vancouver, 1997, Ed. by M. Comyn, M. K. Craddok, M. Reiser, and J. Thomson, Vol. 3, p. 2820.

5. A. V. Agafonov, V. P. Tarakanov, and V. M. Fedorov, Vopr. At. Nauki Tekh., Ser.: Yad.-Fiz. Issled. 1, 134 (1997).

6. Y. M. Saveliev, W. Sibbett, and D. M. Parkes, Phys. Plasmas 4, 2319 (1997).

7. G. I. Churyumov, Izv. Vyssh. Uchebn. Zaved., Radioele-ktron., No. 7, 77(1997).

8. A. I. Vishnevskii, A. I. Soldatenko, and A. I. Shendakov, Izv. Vyssh. Uchebn. Zaved., Radioelektron. 11, 555 (1968).

9. H. Knoepfel, Pulsed High Magnetic Fields (North-Holland, Amsterdam, 1970; Mir, Moscow, 1972).
10. I. I. Kifer, Ferromagnetic Material Tests (Gosenergoiz-dat, Moscow, 1962).

11. V. V. Zakutin and A. M. Shenderovich, Prib. Tekh. Eksp., No. 5, 238 (1976).

12. A. N. Dovbnya, V. V. Zakutin, N. G. Reshetnyak, et al., Vopr. At. Nauki Tekh., Ser.: Yad.-Fiz. Issled. 1, 53 (1997).

13. Yu. Ya. Volkolupov, A. N. Dovbnya, V. V. Zakutin, et al., Zh. Tekh. Fiz. 71 (2), 98 (2001) [Tech. Phys. 46, 227 (2001) (in press)].

14. N. I. Aizatskii, A. N. Dovbnya, V. V. Mitrochenko, et al., Vopr. At. Nauki Tekh., Ser.: Yad.-Fiz. Issled. 3, 38 (1999). 